\title{
Pacific
}

Journal of

Mathematics

\section{INTEGRAL SPINOR NORMS IN DYADIC LOCAL FIELDS. I}

FEI XU

Volume 157 No. 1 


\title{
INTEGRAL SPINOR NORMS \\ IN DYADIC LOCAL FIELDS I
}

\section{FEI Xu}

\begin{abstract}
The spinor norms of integral rotations on some quadratic forms over an arbitrary dyadic local field are determined. As an application, the results obtained in Bon Durant's paper are improved.
\end{abstract}

1. Introduction. Because of the absence of a local-global principle in the equivalence of integral quadratic forms over a global field, Eichler and Kneser developed the theory of spinor genera, which strongly depend on spinor norms of local integral rotations. For nondyadic cases the spinor norms are well understood (see [6]), but they become difficult to be determined when 2 is no longer a unit. Hsia [5], the author [10] computed the spinor norms of local integral rotations on modular quadratic forms completely. Earnest and Hsia [3] determined the spinor norms of integral rotations on arbitrary quadratic forms over a dyadic local field in which 2 is prime, and Bon Durant [1] recently considered the spinor norms of integral rotations over a local field which is a quadratic ramified extension field of $Q_{2}$. In the present article we first determine the spinor norms of all binary quadratic forms over an arbitrary dyadic local field, and then use these results to obtain the spinor norms of some quadratic forms over an arbitrary dyadic local field which Jordan components are one dimension. As an application, we consider the spinor norms of quadratic forms over the dyadic local fields which ramification index of 2 is 2 and improve the sufficient condition for the class number of an indefinite quadratic form over the ring of integers of a number field to be a divisor of the class number of the field.

Notation and terminology used here is that of [8]. In particular, $F$ denotes a dyadic local field, $\vartheta$ the ring of integers in $F, p$ the maximal ideal of $\vartheta, U$ the group of units in $\vartheta, e=$ ord 2 the ramification index of 2 in $F, \pi$ a fixed prime element in $F, D($, the quadratic defect function, $\Delta$ a fixed unit of quadratic defect $4 \vartheta$, $V$ a regular quadratic space over $F$ associated symmetric bilinear form $B(x, y), L$ a lattice on $V, O^{+}(V)$ the group of rotations on $V, O^{+}(L)$ the corresponding subgroup of units of $L$, and $\theta($,$) the$ 
spinor norm function. We use the symbol $\langle a, b, c, \ldots\rangle$ for lattices, and $[a, b, c, \ldots]$ for spaces.

2. Binary cases. In this section we determine the spinor norms of local integral rotations on binary lattices completely. All the results are expressed in convenient closed forms. Some cases have already been treated in [4], but we may repeat them for completeness.

Let $L$ be a binary lattice. If $L$ is modular then $\theta\left(O^{+}(L)\right)$ has been determined in [5] and [10]. So we only consider the nonmodular cases. Since the spinor norm is not affected by scaling, we can put $L=\vartheta x \perp \vartheta y$ with $Q(x)=1$ and $Q(y)=\varepsilon \pi^{r}$ where $r \geq 1$ and $\varepsilon \in U$.

Proposition 2.1. If $r>4 e$, then $\theta\left(O^{+}(L)\right)=\dot{F}^{2} \cup \varepsilon \pi^{r} \dot{F}^{2}$.

Proof. Take a symmetry $\tau_{z}$ in $O(L)$ where $z=a x+b y$ with $a, b \in \vartheta$ and one of them is in $U$. By [4, Prop. 3.2], we get ord $a \leq e$; or ord $a \geq r-e$, ord $b=0$, so $Q(x)$ is in $\dot{F}^{2}$ or $\varepsilon \pi^{r} \dot{F}^{2}$ respectively by the Local Square Theorem, and $\theta\left(O^{+}(L)\right)=\dot{F}^{2} \cup \varepsilon \pi^{r} \dot{F}^{2}$ by [7, $1.1]$.

Proposition 2.2. Suppose $2 e<r \leq 4 e$.

(i) When $r$ is odd, $\theta\left(O^{+}(L)\right)=\left(\left(1+p^{r-2 e}\right) \dot{F}^{2} \cup \varepsilon \pi\left(1+p^{r-2 e}\right) \dot{F}^{2}\right) \cap$ $Q(\dot{F} L)$.

(ii) When $r$ is even, $D(\varepsilon)=p^{d}$ with $1 \leq d \leq 2 e-r / 2, \theta\left(O^{+}(L)\right)=$ $\left(\left(1+p^{r-2 e+d}\right) \dot{F}^{2} \cup \varepsilon\left(1+p^{r-2 e+d}\right) \dot{F}^{2}\right) \cap Q(\dot{F} L)$.

(iii) When $r$ is even, $D(\varepsilon)=p^{d}$ with $d>2 e-r / 2$, or $0 ; \theta\left(O^{+}(L)\right)$ $=\left(1+p^{r / 2}\right) \dot{F}^{2} \cup \varepsilon\left(1+p^{r / 2}\right) \dot{F}^{2}$.

Proof. Take a symmetry $\tau_{z}$ in $O(L)$ where $z=a x+b y$ with $a, b \in \vartheta$ and one of them is in $U$. By [4, Prop. 3.2], we obtain ord $a=0$, ord $b \geq 0$; or $0<$ ord $a \leq e$, ord $b=0$; or ord $a \geq r-e$, ord $b=0$.

Case (i). If ord $a \leq e$, then

$$
\operatorname{ord}\left(a^{-2} b^{2} \varepsilon \pi^{r}\right) \geq r-2 \text { ord } a \geq r-2 e .
$$

So

$$
Q(z)=a^{2}\left(1+a^{-2} b^{2} \varepsilon \pi^{r}\right)
$$

is in $\left(1+p^{r-2 e}\right) \dot{F}^{2} \cap Q(\dot{F} L)$. 
If ord $a \geq r-e$, ord $b=0$; then

$$
\operatorname{ord}\left(\varepsilon^{-1} \pi^{-r}\left(b^{-1} a\right)^{2}\right)=2 \text { ord } a-r \geq r-2 e .
$$

So

$$
Q(z)=\varepsilon \pi^{r} b^{2}\left(1+\varepsilon^{-1} \pi^{-r}\left(b^{-1} a\right)^{2}\right)
$$

is in $\varepsilon \pi\left(1+p^{r-2 e}\right) \dot{F}^{2} \cap Q(\dot{F} L)$. Therefore

$$
\theta\left(O^{+}(L)\right) \subseteq\left(\left(1+p^{r-2 e}\right) \dot{F}^{2} \cup \varepsilon \pi\left(1+p^{r-2 e}\right) \dot{F}^{2}\right) \cap Q(\dot{F} L)
$$

by $[7,1.1]$.

Conversely, take an element $h$ in $\left(1+p^{r-2 e}\right) \dot{F}^{2} \cap Q(\dot{F} L)$, so there exists $z$ in $F L$ such that $Q(z)=h$. Without loss of generality, we assume $z=a x+b y$ where $a, b \in \vartheta$ and one of them is a unit.

If $e<$ ord $a<r / 2$, then ord $b=0$. Put

$$
k=\operatorname{ord}\left(\varepsilon \pi^{r}\left(b a^{-1}\right)^{2}\right)=r-2 \text { ord } a<r-2 e \leq 2 e .
$$

Note that $k$ is a positive odd integer. Since $h$ is in $\left(1+p^{r-2 e}\right) \dot{F}^{2}$, we obtain

$$
\left(1+c \pi^{r-2 e}\right) f^{2}=h=a^{2}+\varepsilon \pi^{r} b^{2}=a^{2}\left(1+\left(a^{-1} b\right)^{2} \varepsilon \pi^{r}\right)
$$

where $f \in \dot{F}^{2}, c \in \vartheta$. Let $\eta=a f^{-1}$, so $\eta$ is in $U$ by the above equation, and

$$
\eta^{2}\left(1+\varepsilon \pi^{r}\left(b a^{-1}\right)^{2}\right)=\left(1+c \pi^{r-2 e}\right) .
$$

But

$$
D\left(\eta^{2}\left(1+\varepsilon \pi^{r}\left(b a^{-1}\right)^{2}\right)=p^{k} \supset p^{r-2 e} \supseteq D\left(1+c \pi^{r-2 e}\right)\right.
$$

by $[8,63: 5]$, a contradiction.

If $r-e>$ ord $a>r / 2$, then ord $b=0$ again,

$$
\text { ord } h=\operatorname{ord}\left(a^{2}+\varepsilon \pi^{r} b^{2}\right)=r
$$

is odd. But $h$ is in $\left(1+p^{r-2 e}\right) \dot{F}^{2}$, so that ord $h$ is even. This is a contradiction. Now there remains ord $a=0$; or $0<$ ord $a \leq e$, ord $b=0$; or ord $a \geq r-e$, ord $b=0$, so $\tau_{z}$ is in $O(L)$ by [4, Prop. 3.2]. Therefore

$$
\theta\left(O^{+}(L)\right)=\left(\left(1+p^{r-2 e}\right) \cup \varepsilon \pi\left(1+p^{r-2 e}\right) \dot{F}^{2}\right) \cap Q(\dot{F} L) .
$$

Case (ii). Since $D(\varepsilon)=p^{d}$, we can assume $\varepsilon=1+\sigma \pi^{d}$ for convenience, where $\sigma \in U$ and $d$ is odd. Note $1 \leq d \leq 2 e-r / 2<2 e$.

If ord $a=0$, then

$$
\operatorname{ord}\left(\varepsilon \pi^{r}\left(a^{-1} b\right)^{2}\right)=r+2 \text { ord } b \geq r>r-2 e+d .
$$


So

$$
Q(z)=a^{2}\left(1+\varepsilon \pi^{r}\left(a^{-1} b\right)^{2}\right)
$$

is in $\left(1+p^{r-2 e}\right) \dot{F}^{2} \cap Q(\dot{F} L)$. If $0<$ ord $a \leq e<r / 2$, ord $b=0$ then

$$
\operatorname{ord}\left(\sigma \pi^{d+r}\left(a^{-1} b\right)^{2}\right)=r+d-2 \text { ord } a \geq r+d-2 e
$$

and

$$
\operatorname{ord}\left(-2 \pi^{r / 2}\left(a^{-1} b\right)\right)=e+r / 2-\text { ord } a \geq r / 2 \geq r+d-2 e .
$$

So

$$
\begin{aligned}
Q(z) & =a^{2}\left(1+\varepsilon \pi^{r}\left(a^{-1} b\right)^{2}\right)=a^{2}\left(1+\left(1+\sigma \pi^{d}\right) \pi^{r}\left(a^{-1} b\right)^{2}\right) \\
& =a^{2}\left(1+\pi^{r}\left(a^{-1} b\right)^{2}+\sigma \pi^{r+d}\left(a^{-1} b\right)^{2}\right) \\
& =a^{2}\left(\left(1+\pi^{r / 2}\right)^{2}-2 \pi^{r / 2}\left(a^{-1} b\right)+\sigma \pi^{d+r}\left(a^{-1} b\right)^{2}\right)
\end{aligned}
$$

is in $\left(1+p^{r-2 e+d}\right) \dot{F}^{2} \cap Q(\dot{F} L)$.

If ord $a \geq r-e$, ord $b=0$; then

$$
\operatorname{ord}\left(-2\left(\varepsilon^{-1} b^{-1}\right) a \pi^{-r / 2}\right)=e+\text { ord } a-r / 2 \geq r-2 e+d
$$

and

$$
\operatorname{ord}\left(\sigma\left(\varepsilon^{-1} b^{-1}\right)^{2} a^{2} \pi^{-r+d}\right)=2 \text { ord } a-r+d \geq r-2 e+d .
$$

So

$$
\begin{aligned}
Q(z) & =\varepsilon \pi^{r} b^{2}\left(1+\varepsilon\left(\varepsilon^{-1} b^{-1}\right)^{2} a^{2} \pi^{-r}\right) \\
& =\varepsilon \pi^{r} b^{2}\left(1+\left(1+\sigma \pi^{d}\right)\left(\varepsilon^{-1} b^{-1}\right)^{2} a^{2} \pi^{-r}\right) \\
& =\varepsilon \pi^{r} b^{2}\left(1+\left(\varepsilon^{-1} b^{-1}\right)^{2} a^{2} \pi^{-r}+\sigma\left(\varepsilon^{-1} b^{-1}\right)^{2} a^{2} \pi^{-r+d}\right) \\
= & \varepsilon \pi^{r} b^{2}\left(\left(1+\varepsilon^{-1} b^{-1} a \pi^{-r / 2}\right)^{2}-2 \varepsilon^{-1} b^{-1} a \pi^{-r / 2}\right. \\
& \left.\quad+\sigma\left(\varepsilon^{-1} b^{-1}\right)^{2} a^{2} \pi^{-r+d}\right)
\end{aligned}
$$

is in $\varepsilon\left(1+p^{r-2 e+d}\right) \dot{F}^{2} \cap Q(\dot{F} L)$. Therefore

$$
\theta\left(O^{+}(L)\right) \subseteq\left(\left(1+p^{r-2 e+d}\right) \dot{F}^{2} \cup \varepsilon\left(1+p^{r-2 e+d}\right) \dot{F}^{2}\right) \cap Q(\dot{F} L) .
$$

Conversely, take an element $h$ in $\left(1+p^{r-2 e+d}\right) \dot{F}^{2} \cap Q(\dot{F} L)$, so there exists $z$ in $F L$ such that $Q(z)=h$. Without loss of generality, we assume $z=a x+b y$ where $a, b \in \vartheta$ and one of them is a unit.

If $e<$ ord $a<r / 2$, then ord $b=0$. Put

$$
\begin{aligned}
k & =\operatorname{ord}\left(\sigma\left(a^{-1} b\right)^{2} \pi^{r+d}\right)=r+d-2 \operatorname{ord} a, \\
& =(e+r / 2-\operatorname{ord} a)+(r / 2+d-e-\operatorname{ord} a) \\
& <\min \{(e+r / 2-\operatorname{ord} a)+(r / 2+d-2 e), r+d-2 e\} \\
& \leq \min \{(e+r / 2-\operatorname{ord} a), r+d-2 e\} \\
& =\min \left\{\operatorname{ord}\left(-2 a^{-1} b \pi^{r / 2}\right), r+d-2 e\right\}
\end{aligned}
$$


and $k$ is a positive odd integer. Since $h$ is in $\left(1+p^{r-2 e+d}\right) \dot{F}^{2}$, we obtain

$$
f^{2}\left(1+c \pi^{r-2 e+d}\right)=h=\left(a^{2}+\varepsilon \pi^{r} b^{2}\right)=a^{2}\left(1+\left(a^{-1} b\right)^{2} \varepsilon \pi^{r}\right)
$$

where $f \in \dot{F}, c \in \vartheta$. Let $\eta=a f^{-1}$, so $\eta$ is in $U$ by the above equation, and

$$
\begin{aligned}
1+c \pi^{r-2 e+d} & =\eta^{2}\left(1+\varepsilon \pi^{r}\left(a^{-1} b\right)^{2}\right) \\
& =\eta^{2}\left(1+\left(1+\sigma \pi^{d}\right) \pi^{r}\left(a^{-1} b\right)^{2}\right) \\
& =\eta^{2}\left(1+\pi^{r}\left(a^{-1} b\right)^{2}+\sigma \pi^{r+d}\left(a^{-1} b\right)^{2}\right) \\
& =\eta^{2}\left(\left(1+\left(a^{-1} b\right) \pi^{r / 2}\right)^{2}-2\left(a^{-1} b\right) \pi^{r / 2}+\sigma \pi^{r+d}\left(a^{-1} b\right)^{2}\right) .
\end{aligned}
$$

But

$$
\begin{gathered}
D\left(\eta^{2}\left(\left(1+a^{-1} b \pi^{r / 2}\right)^{2}-2\left(a^{-1} b\right) \pi^{r / 2}+\sigma \pi^{r+d}\left(a^{-1} b\right)^{2}\right)\right) \\
\quad=p^{k} \supset p^{r+d-2 e} \supseteq D\left(1+c \pi^{r-2 e+d}\right)
\end{gathered}
$$

by $[8,63: 5]$, a contradiction.

If ord $a=r / 2$, then ord $b=0$. Let $\xi=a^{-1} \pi^{r / 2} b$, so $\xi$ is in $U$. Suppose

$$
\begin{aligned}
h & =a^{2}\left(1+\varepsilon \pi^{r}\left(a^{-1} b\right)^{2}\right)=a^{2}\left(1+\varepsilon \xi^{2}\right)=a^{2}\left(1+\left(1+\sigma \pi^{d}\right) \xi^{2}\right) \\
& =a^{2}\left(1+\xi^{2}+\sigma \xi^{2} \pi^{d}\right)=a^{2}\left((1+\xi)^{2}-2 \xi+\sigma \xi^{2} \pi^{d}\right)
\end{aligned}
$$

is in $\left(1+p^{r-2 e+d}\right) \dot{F}^{2}$. Note $d \leq 2 e-r / 2<e$, and $d$ is odd, so $2 \operatorname{ord}(1+\xi)=\operatorname{ord}(1+\xi)^{2}<d<e$.

Writing $1+\xi=\delta \pi^{s}$ with $\delta \in U, s \geq 0$, we obtain

$$
\eta^{2}\left(1-\delta^{-2} \xi 2 \pi^{-2 s}+\sigma \delta^{-2} \xi^{2} \pi^{d-2 s}\right)=\left(1+c \pi^{r-2 e+d}\right)
$$

where $\eta \in U, c \in \vartheta$. But

$$
\begin{aligned}
& D\left(\eta^{2}\left(1-\delta^{-2} \xi 2 \pi^{-2 s}+\sigma \delta^{-2} \xi^{2} \pi^{d-2 s}\right)\right) \\
& \quad=p^{d-2 s} \supseteq p^{d} \supset p^{r-2 e+d} \supseteq D\left(1+c \pi^{r-2 e+d}\right)
\end{aligned}
$$

by [8, 63:5], a contradiction

If $r / 2<$ ord $a<r-e$, then ord $b=0$. We have

$$
\operatorname{ord}\left(2 b^{-1} a \pi^{-r / 2}\right)=e+\text { ord } a-r / 2>e>2 e-r / 2 \geq d \geq 1,
$$

and

$$
\begin{aligned}
h & =Q(z)=b^{2} \pi^{r}\left(\varepsilon+\left(b^{-1} a \pi^{-r / 2}\right)^{2}\right)=b^{2} \pi^{r}\left(1+\sigma \pi^{d}+\left(b^{-1} a \pi^{-r / 2}\right)^{2}\right) \\
& =b^{2} \pi^{r}\left(\left(1+b^{-1} a \pi^{-r / 2}\right)^{2}-2 b^{-1} a \pi^{-r / 2}+\sigma \pi^{d}\right)
\end{aligned}
$$

is in $\left(1+p^{r-2 e+d}\right) \dot{F}^{2}$ by hypothesis. So

$$
\eta^{2}\left(\left(1+b^{-1} a \pi^{-r / 2}\right)^{2}-2 b^{-1} a \pi^{-r / 2}+\sigma \pi^{d}\right)=\left(1+c \pi^{r-2 e+d}\right)
$$


where $\eta \in U$ and $c \in \vartheta$. But

$$
\begin{gathered}
D\left(\eta^{2}\left(\left(1+b^{-1} a\left(\pi^{-r / 2}\right)^{2}-2 b^{-1} a \pi^{-r / 2}+\sigma \pi^{d}\right)\right)\right. \\
\quad=p^{d} \supset p^{r-2 e+d} \supseteq D\left(1+c \pi^{r-2 e+d}\right)
\end{gathered}
$$

by $[8,63: 5]$, a contradiction.

Now there remains ord $a=0$ or $0<$ ord $a \leq e$, ord $b=0$; or ord $a \geq r-e$, ord $b=0$, so $\tau_{z}$ is in $O(L)$ by [4, Prop. 3.2]. Therefore

$$
\theta\left(O^{+}(L)\right)=\left(\left(1+p^{r-2 e+d}\right) \dot{F}^{2} \cup \varepsilon\left(1+p^{r-2 e+d}\right) \dot{F}^{2}\right) \cap Q(\dot{F} L) .
$$

Case (iii). W'rite $\varepsilon=1+\sigma \pi^{d}$ with $d>2 e-r / 2, \sigma \in U$.

If ord $a=0$, then

$$
\operatorname{ord}\left(\varepsilon \pi^{r}\left(a^{-1} b\right)^{2}\right)=r+2 \text { ord } b \geq r \geq r / 2,
$$

so

$$
Q(z)=a^{2}\left(1+\varepsilon \pi^{r}\left(a^{-1} b\right)^{2}\right)
$$

is in $\left(1+p^{r / 2}\right) \dot{F}^{2}$.

If $0<$ ord $a \leq e<r / 2$, ord $b=0$; then

$$
\begin{aligned}
r / 2 & \leq \operatorname{ord}\left(-2\left(a^{-1} b\right) \pi^{r / 2}\right)=e-\operatorname{ord} a+r / 2 \\
& =(r+d-2 \operatorname{ord} a)+(\operatorname{ord} a-r / 2-d+e) \\
& \leq(r+d-2 \operatorname{ord} a)+(2 e-r / 2-d) \\
& <r+d-2 \operatorname{ord} a=\operatorname{ord}\left(\sigma \pi^{r+d}\left(a^{-1} b\right)^{2}\right) .
\end{aligned}
$$

So

$$
\begin{aligned}
Q(z) & =a^{2}\left(1+\varepsilon \pi^{r}\left(a^{-1} b\right)^{2}\right)=a^{2}\left(1+\left(1+\sigma \pi^{d}\right) \pi^{r}\left(a^{-1} b\right)^{2}\right) \\
& =a^{2}\left(1+\left(a^{-1} b\right)^{2} \pi^{r}+\sigma\left(a^{-1} b\right)^{2} \pi^{r+d}\right) \\
& \left.=a^{2}\left(1+a^{-1} b \pi^{r / 2}\right)^{2}-2 a^{-1} b \pi^{r / 2}+\sigma\left(a^{-1} b\right)^{2} \pi^{r+d}\right)
\end{aligned}
$$

is in $\left(1+p^{r / 2}\right) \dot{F}^{2}$.

If ord $a \geq r-e>r / 2$, ord $b=0$; then

$$
\begin{aligned}
r / 2 & \leq \operatorname{ord}\left(-2 \varepsilon^{-1} b^{-1} a \pi^{-r / 2}\right)=e+\text { ord } a-r / 2 \\
& =(2 \text { ord } a+d-r)+(r / 2-\text { ord } a+e-d) \\
& \leq(2 \text { ord } a+d-r)+(2 e-r / 2-d) \\
& <(2 \text { ord } a+d-r)=\operatorname{ord}\left(\sigma\left(\varepsilon^{-1} b^{-1} a\right)^{2} \pi^{d-r}\right) .
\end{aligned}
$$


So

$$
\begin{aligned}
Q(z) & =\varepsilon \pi^{r} b^{2}\left(1+\varepsilon\left(\varepsilon^{-1} b^{-1} a\right)^{2} \pi^{-r}\right) \\
= & \varepsilon \pi^{r} b^{2}\left(1+\left(1+\sigma \pi^{d}\right)\left(\varepsilon^{-1} b^{-1} a\right)^{2} \pi^{-r}\right) \\
= & \varepsilon \pi^{r} b^{2}\left(1+\left(\varepsilon^{-1} b^{-1} a\right)^{2} \pi^{-r}+\sigma\left(\varepsilon^{-1} b^{-1} a\right)^{2} \pi^{d-r}\right) \\
= & \varepsilon \pi^{r} b^{2}\left(\left(1+\varepsilon^{-1} b^{-1} a \pi^{-r / 2}\right)^{2}-2 \varepsilon^{-1} b^{-1} a \pi^{-r / 2}\right. \\
& \left.+\sigma\left(\varepsilon^{-1} b^{-1} a\right)^{2} \pi^{d-r}\right)
\end{aligned}
$$

is in $\varepsilon\left(1+p^{r / 2}\right) \dot{F}^{2}$.

Therefore

$$
\theta\left(O^{+}(L)\right) \subseteq\left(1+p^{r / 2}\right) \dot{F}^{2} \cup \varepsilon\left(1+p^{r / 2}\right) \dot{F}^{2}
$$

by $[7,1.1]$.

Conversely, take an element $h$ in $\left(1+p^{r / 2}\right) \dot{F}^{2}$, by the Local Square Theorem, we assume $h=1+c \pi^{r / 2}$ with $0 \leq$ ord $c<e$. Write $c=\lambda \pi^{k}$ with $\lambda \in U$. By Hensel's lemma, there exists $\eta$ in $U$ such that

$$
\left(\pi^{r-2 e+2 k}-\sigma c^{-1} \pi^{d+r / 2-2 e+2 k}\right) \eta^{2}\left(2 c^{-1} \pi^{k-e}+2 \pi^{r / 2-e+k}\right) \eta+1=0 .
$$

Put $z=\pi^{e-k} x+\eta y$, so $\tau_{z}$ is in $O(L)$ by [4, Prop. 3.2], and

$$
\begin{aligned}
Q(z)= & \pi^{2 e-2 k}+\varepsilon \pi^{r} \eta^{2}=\pi^{2 e-2 k}+\left(1+\sigma \pi^{d}\right) \pi^{r} \eta^{2} \\
= & \pi^{2 e-2 k}\left(1+\pi^{r+2 k-2 e} \eta^{2}+\sigma \pi^{d+r+2 k-2 e} \eta^{2}\right) \\
= & \pi^{2 e-2 k}\left(\left(1+\pi^{r / 2+k-e} \eta\right)^{2}-2 \pi^{r / 2+k-e} \eta+\sigma \pi^{d+r+2 k-2 e} \eta^{2}\right) \\
= & \pi^{2 e-2 k}\left(\left(1+\pi^{r / 2+k-e} \eta\right)^{2}\right. \\
& \left.\quad+\left(-2 c^{-1} \pi^{k-e} \eta+\sigma c^{-1} \pi^{d+r / 2+2 k-2 e} \eta^{2}\right) c \pi^{r / 2}\right) \\
= & \pi^{2 e-2 k}\left(\left(1+\pi^{r / 2+k-e} \eta\right)^{2}+\left(1+\pi^{r / 2-e+k} \eta\right)^{2} c \pi^{r / 2}\right) \\
= & \pi^{2 e-2 k}\left(1+\pi^{r / 2+k-e} \eta\right)^{2}\left(1+c \pi^{r / 2}\right)=\pi^{2 e-2 k}\left(1+\pi^{r / 2+k-e} \eta\right)^{2} h .
\end{aligned}
$$

Therefore

$$
\theta\left(O^{+}(L)\right)=\left(1+p^{r / 2}\right) \dot{F}^{2} \cup \varepsilon\left(1+p^{r / 2}\right) \dot{F}^{2} .
$$

Proposition 2.3. Suppose $0<r \leq 2 e$.

(i) When $r$ is odd, or $r$ is even and $D(-\varepsilon)=p^{d}$ with $1 \leq d \leq$ $e-r / 2 ; \theta\left(O^{+}(L)\right)=Q(\dot{F} L)$.

(ii) When $r$ is even and $D(-\varepsilon)=p^{d}$ with $(3 e-r / 2) / 2 \geq d>$ $e-r / 2 ; \theta\left(O^{+}(L)\right)=\left(\left(1+p^{d-e+r / 2}\right) \dot{F}^{2}\right) \cap Q(\dot{F} L)$.

(iii) When $r$ is even and $D(-\varepsilon)=p^{d}$ with $d>(3 e-r / 2) / 2$, or 0 ; $\theta\left(O^{+}(L)\right)=\left(1+p^{e-[e / 2-r / 4]}\right) \dot{F}^{2}$. 
Proof. Take a maximal anisotorpic vector $z=a x+b y$ in $L$, where $a, b \in \vartheta$ and one of them is a unit, we know $\tau_{z}$ is in $O(L)$ if and only if $2 B(z, L) \subseteq Q(z) \vartheta$. This is equivalent to

$$
\operatorname{ord}\left(a^{2}+\varepsilon \pi^{r} b^{2}\right) \leq \min \{\text { ord } a, r+\text { ord } b\}+e .
$$

In fact, this inequality is the same as ord $a \neq r / 2$; or ord $a=r / 2$, ord $b=0,0 \leq \operatorname{ord}\left(\left(a \pi^{-r / 2} b^{-1}\right)^{2}+\varepsilon\right) \leq e-r / 2$.

Case (i). When $r$ is odd, we always have ord $a \neq r / 2$; when $r$ is even and ord $a=r / 2$, we have $0 \leq \operatorname{ord}\left(\varepsilon+\left(\pi^{-r / 2} b^{-1} a\right)^{2}\right) \leq d \leq$ $e-r / 2$ since $D(-\varepsilon)=p^{d}$ with $1 \leq d \leq e-r / 2$. Note $F L$ is anisotropic in this case, so $\theta\left(O^{+}(L)\right)=Q(\dot{F} L)$.

Case (ii). Write $-\varepsilon=1+\sigma \pi^{d}$ with $\sigma \in U$ and $2 e>(3 e-r / 2) / 2 \geq$ $d>e-r / 2$.

If ord $a=0$, then

$$
\begin{aligned}
& \operatorname{ord}\left(\sigma\left(\pi^{r / 2} b a^{-1}\right)^{2} \pi^{d}\right)=d+r+2 \operatorname{ord} b \\
& \quad=(e+r / 2+\operatorname{ord} b)+\operatorname{ord} b+(d+r / 2-e) \\
& \quad>e+r / 2+\operatorname{ord} b=\operatorname{ord}\left(-2 \pi^{r / 2}\left(b a^{-1}\right)\left(1+a^{-1} b \pi^{r / 2}\right)\right) \\
& \quad \geq e+r / 2>d-e+r / 2 .
\end{aligned}
$$

So

$$
\begin{aligned}
Q(z)= & a^{2}\left(1+\varepsilon \pi^{r}\left(a^{-1} b\right)^{2}\right)=a^{2}\left(1-\left(1+\sigma \pi^{d}\right)\left(a^{-1} b\right)^{2} \pi^{r}\right) \\
= & a^{2}\left(\left(1+a^{-1} b \pi^{r / 2}\right)^{2}\right. \\
& \left.-2\left(a^{-1} b \pi^{r / 2}\right)\left(1+a^{-1} b \pi^{r / 2}\right)-\sigma\left(a^{-1} b \pi^{r / 2}\right)^{2} \pi^{d}\right)
\end{aligned}
$$

is in $\left(1+p^{d-e+r / 2}\right) \dot{F}^{2} \cap Q(\dot{F} L)$.

If $0<$ ord $a<r / 2$, ord $b=0$; then

$$
\begin{aligned}
& \operatorname{ord}\left(-2 a^{-1} b \pi^{r / 2}\left(1+a^{-1} b \pi^{r / 2}\right)\right)=e+r / 2-\text { ord } a \\
& \quad>e=(e+e / 2-r / 4)+r / 4-e / 2 \geq d+(r / 2-e) / 2 \\
& \quad \geq d-e+r / 2
\end{aligned}
$$

and

$$
\operatorname{ord}\left(-\sigma\left(a^{-1} b \pi^{r / 2}\right)^{2} \pi^{d}\right)=d+r-2 \text { ord } a \geq d \geq d-e+r / 2 .
$$

So

$$
\begin{aligned}
Q(z)=a^{2}( & \left(1+a^{-1} b \pi^{r / 2}\right)^{2} \\
& \left.-2\left(a^{-1} b \pi^{r / 2}\right)\left(1+a^{-1} b \pi^{r / 2}\right)-\sigma\left(a^{-1} b \pi^{r / 2}\right)^{2} \pi^{d}\right)
\end{aligned}
$$

is in $\left(1+p^{d-e+r / 2}\right) \dot{F}^{2} \cap Q(\dot{F} L)$. 
If ord $a>r / 2$, ord $b=0$; then

$$
\begin{array}{r}
\operatorname{ord}\left(-2 \varepsilon^{-1} b^{-1} a \pi^{-r / 2}\left(1+\varepsilon^{-1} b^{-1} a \pi^{-r / 2}\right)\right) \\
=e+\operatorname{ord} a-r / 2>e \geq d-e+r / 2
\end{array}
$$

and

$$
\operatorname{ord}\left(-\sigma\left(\varepsilon^{-1} b^{-1} a \pi^{-r / 2}\right)^{2} \pi^{d}\right)=-r+2 \text { ord } a+d>d \geq d-e+r / 2 .
$$

So

$$
\begin{aligned}
& Q(z)= \varepsilon \pi^{r} b^{2}\left(1+\varepsilon\left(\varepsilon^{-1} b^{-1} a \pi^{-r / 2}\right)^{2}\right) \\
&=\varepsilon \pi^{r} b^{2}\left(1-\left(1+\sigma \pi^{d}\right)\left(\varepsilon^{-1} b^{-1} a \pi^{-r / 2}\right)^{2}\right) \\
&=\varepsilon \pi^{r} b^{2}\left(\left(1+\varepsilon^{-1} b^{-1} a \pi^{-r / 2}\right)^{2}\right. \\
& \quad-2 \varepsilon^{-1} b^{-1} a \pi^{-r / 2}\left(1+\varepsilon^{-1} b^{-1} a \pi^{-r / 2}\right) \\
&\left.\quad-\sigma\left(\varepsilon^{-1} b^{-1} a \pi^{-r / 2}\right)^{2} \pi^{d}\right)
\end{aligned}
$$

is in $\varepsilon\left(1+p^{d-e+r / 2}\right) \dot{F}^{2} \cap Q(\dot{F} L)$.

If ord $a=r / 2$, ord $b=0$, and $\operatorname{ord}\left(\varepsilon+\left(\pi^{-r / 2} a b^{-1}\right)^{2}\right) \leq e-r / 2$, note

$$
\operatorname{ord}\left(2 a^{-1} b \pi^{r / 2}\left(1+a^{-1} b \pi^{r / 2}\right)\right)=e+\operatorname{ord}\left(1+a^{-1} b \pi^{r / 2}\right) \geq e>e-r / 2
$$

and

$$
\operatorname{ord}\left(\sigma\left(a^{-1} b \pi^{r / 2}\right)^{2} \pi^{d}\right)=d>e-r / 2
$$

Then

$$
\begin{aligned}
\operatorname{ord}(1+ & \left.a^{-1} b \pi^{r / 2}\right)^{2} \\
= & \operatorname{ord}\left(\left(1+a^{-1} b \pi^{r / 2}\right)^{2}\right. \\
& -2 a^{-1} b \pi^{r / 2}\left(1+a^{-1} b \pi^{r / 2}\right)^{2}-\sigma\left(a^{-1} b \pi^{r / 2}\right)^{2} \pi^{d} \\
& \left.+2 a^{-1} b \pi^{r / 2}\left(1+a^{-1} b \pi^{r / 2}\right)+\sigma\left(a^{-1} b \pi^{r / 2}\right)^{2} \pi^{d}\right) \\
= & \operatorname{ord}\left(1-\left(a^{-1} b \pi^{r / 2}\right)^{2}-\sigma\left(a^{-1} b \pi^{r / 2}\right)^{2} \pi^{d}\right. \\
& \left.\quad+2 a^{-1} b \pi^{r / 2}\left(1+a^{-1} b \pi^{r / 2}\right)+\sigma\left(a^{-1} b \pi^{r / 2}\right)^{2} \pi^{d}\right) \\
= & \operatorname{ord}\left(1+\left(a^{-1} b \pi^{r / 2}\right)^{2}\left(-1-\sigma \pi^{d}\right)\right. \\
& \left.+2 a^{-1} b \pi^{r / 2}\left(1+a^{-1} b \pi^{r / 2}\right)+\sigma\left(a^{-1} b \pi^{r / 2}\right)^{2} \pi^{d}\right) \\
= & \operatorname{ord}\left(\left(1+\varepsilon\left(a^{-1} b \pi^{r / 2}\right)^{2}\right)\right. \\
& \left.+2 a^{-1} b \pi^{r / 2}\left(1+a^{-1} b \pi^{r / 2}\right)+\sigma\left(a^{-1} b \pi^{r / 2}\right)^{2} \pi^{d}\right) \\
= & \operatorname{ord}\left(1+\varepsilon\left(a^{-1} b \pi^{r / 2}\right)^{2}\right) \leq e-r / 2 .
\end{aligned}
$$


So

$$
\begin{aligned}
& \operatorname{ord}\left(\sigma \pi^{d}\left(a^{-1} b \pi^{r / 2}\right)^{2}\left(1+a^{-1} b \pi^{r / 2}\right)^{-2}\right) \\
& \quad=d-2 \operatorname{ord}\left(1+a^{-1} b \pi^{r / 2}\right) \geq d-e+r / 2
\end{aligned}
$$

and

$$
\begin{aligned}
& \operatorname{ord}\left(\left(-2 a^{-1} b \pi^{r / 2}\right)\left(1+a^{-1} b \pi^{r / 2}\right)^{-1}\right)=e-\operatorname{ord}\left(1+a^{-1} b \pi^{r / 2}\right) \\
& \quad \geq e-(e-r / 2) / 2 \geq d-e+r / 2 .
\end{aligned}
$$

Thus

$$
\begin{aligned}
Q(z)= & a^{2}\left(1+\left(a^{-1} b \pi^{r / 2}\right) \varepsilon\right)=a^{2}\left(1-\left(1+\sigma \pi^{d}\right)\left(a^{-1} b \pi^{r / 2}\right)^{2}\right) \\
= & a^{2}\left(\left(1+a^{-1} b \pi^{r / 2}\right)^{2}\right. \\
& \left.\quad-2\left(1+a^{-1} b \pi^{r / 2}\right) a^{-1} b \pi^{r / 2}-\sigma\left(a^{-1} b \pi^{r / 2}\right)^{2} \pi^{d}\right) \\
= & a^{2}\left(1+a^{-1} b \pi^{r / 2}\right)^{2}\left(1-2 a^{-1} b \pi^{r / 2}\left(1+a^{-1} b \pi^{r / 2}\right)^{-1}\right. \\
& \left.\quad-\sigma \pi^{d}\left(a^{-1} b \pi^{r / 2}\right)^{2}\left(1+a^{-1} b \pi^{r / 2}\right)^{-2}\right)
\end{aligned}
$$

is in $\left(1+p^{d-e+r / 2}\right) \dot{F}^{2} \cap Q(\dot{F} L)$. Therefore

$$
\theta\left(O^{+}(L)\right) \subseteq\left(\left(1+p^{d-e+r / 2}\right) \dot{F}^{2} \cap \varepsilon\left(1+p^{d-e+r / 2}\right) \dot{F}^{2}\right) \cap Q(\dot{F} L) .
$$

Conversely, take an element $h$ in $\left(1+p^{d-e+r / 2}\right) \dot{F}^{2} \cap Q(\dot{F} L)$, so there exists $z$ in $\dot{F} L$ such that $h=Q(z)$. We can assume $z=a x+b y$ with $a, b \in \vartheta$ and one of them is in $U$. We claim that $\tau_{z}$ is in $O(L)$. In fact, if ord $a=r / 2$, ord $b=0$, and $\operatorname{ord}\left(\left(\pi^{-r / 2} a b^{-1}\right)^{2}+\varepsilon\right)>e-r / 2$; note

$$
\begin{aligned}
h & =Q(z)=a^{2}\left(1+\varepsilon\left(a^{-1} b \pi^{r / 2}\right)^{2}\right)=a^{2}\left(1+\left(-1-\sigma \pi^{d}\right)\left(a^{-1} b \pi^{r / 2}\right)^{2}\right) \\
& =a^{2}\left(\left(1+a^{-1} b \pi^{r / 2}\right)^{2}-2 a^{-1} b \pi^{r / 2}\left(1+a^{-1} b \pi^{r / 2}\right)-\sigma\left(a^{-1} b \pi^{r / 2}\right)^{2} \pi^{d}\right)
\end{aligned}
$$

is in $\left(1+p^{d-e+r / 2}\right) \dot{F}^{2}$, and $d$ is odd, $d<2 e$; then

$$
\begin{aligned}
& \operatorname{ord}\left(1+a^{-1} b \pi^{r / 2}\right)^{2} \\
& \quad=\operatorname{ord}\left(\left(1+a^{-1} b \pi^{r / 2}\right)^{2}\right. \\
& \left.\quad \quad-2 a^{-1} b \pi^{r / 2}\left(1+a^{-1} b \pi^{r / 2}\right)-\sigma\left(a^{-1} b \pi^{r / 2}\right)^{2} \pi^{d}\right) \\
& =\operatorname{ord}\left(1+\varepsilon\left(a^{-1} b \pi^{r / 2}\right)^{2}\right)>e-r / 2 .
\end{aligned}
$$

Write $1+a^{-1} b \pi^{r / 2}=\lambda \pi^{s}$ with $\lambda \in U, s \geq 0$. So $d>2 s>e-r / 2$, and

$\eta^{2}\left(1-\left(\lambda^{-1} a^{-1} b \pi^{r / 2}\right)\left(2 \pi^{-s}\right)-\sigma\left(\lambda^{-1} a^{-1} b \pi^{r / 2}\right)^{2} \pi^{d-2 s}\right)=1+c \pi^{d-e+r / 2}$

where $\eta \in U, c \in \vartheta$. Since $d \leq e+(e-r / 2) / 2<e+s$, that is $0<d-2 s<e-s$, and $d-2 s$ is odd, we have

$$
\begin{gathered}
D\left(\eta^{2}\left(1-\left(\lambda^{-1} a^{-1} b \pi^{r / 2}\right)\left(2 \pi^{-s}\right)-\sigma\left(\lambda^{-1} a^{-1} b \pi^{r / 2}\right)^{2} \pi^{d-2 s}\right)\right)=p^{d-2 s} \\
\supset p^{d-e+r / 2} \supseteq D\left(1+c \pi^{d-e+r / 2}\right) .
\end{gathered}
$$


This leads to a contradiction. So $\tau_{z}$ is in $O(L)$, and

$$
\theta\left(O^{+}(L)\right)=\left(\left(1+p^{d-e+r / 2}\right) \dot{F}^{2} \cup \varepsilon\left(1+p^{d-e+r / 2}\right) \dot{F}^{2}\right) \cap Q(\dot{F} L) .
$$

Case (iii). Write $-\varepsilon=1+\sigma \pi^{d}$ with $\sigma \in U$ and $d>(3 e-r / 2) / 2$.

If ord $a=0$, then

$$
\begin{gathered}
\operatorname{ord}\left(-2\left(1+a^{-1} b \pi^{r / 2}\right) a^{-1} b \pi^{r / 2}\right)=e+\operatorname{ord} b+r / 2 \\
\geq e+r / 2>e-[e / 2-r / 4]
\end{gathered}
$$

and

$$
\begin{aligned}
& \operatorname{ord}\left(-\sigma\left(a^{-1} b \pi^{r / 2}\right)^{2} \pi^{d}\right)=2 \text { ord } b+r+d \\
& \quad \geq r+d>d>e+[e / 2-r / 4] \geq e-[e / 2-r / 4] .
\end{aligned}
$$

So

$$
\begin{aligned}
Q(z)= & a^{2}\left(1-\left(a^{-1} b \pi^{r / 2}\right)^{2}\left(1+\sigma \pi^{d}\right)\right) \\
= & a^{2}\left(\left(1+a^{-1} b \pi^{r / 2}\right)^{2}\right. \\
& \left.\quad-2\left(1+a^{-1} b \pi^{r / 2}\right) a^{-1} b \pi^{r / 2}-\sigma\left(a^{-1} b \pi^{r / 2}\right)^{2} \pi^{d}\right)
\end{aligned}
$$

is in $\left(1+p^{e-[e / 2-r / 4]}\right) \dot{F}^{2}$.

If ord $a>r / 2$, ord $b=0$; then

$$
\begin{aligned}
\operatorname{ord}\left(-2\left(\varepsilon^{-1} b^{-1} \pi^{-r / 2} a\right)\left(1+\varepsilon^{-1} b^{-1} \pi^{-r / 2} a\right)\right) \\
\quad=e-r / 2+\text { ord } a>e \geq e-[e / 2-r / 4]
\end{aligned}
$$

and

$$
\begin{gathered}
\operatorname{ord}\left(-\sigma\left(\varepsilon^{-1} b^{-1} \pi^{-r / 2} a\right)^{2} \pi^{d}\right)=d+2 \text { ord } a-r \\
>d>e+[e / 2-r / 4] \geq e-[e / 2-r / 4] .
\end{gathered}
$$

So

$$
\begin{aligned}
Q(z)= & \varepsilon \pi^{r} b^{2}\left(1+\varepsilon\left(\varepsilon^{-1} b^{-1} \pi^{-r / 2} a\right)^{2}\right) \\
= & \varepsilon \pi^{r} b^{2}\left(1-\left(1+\sigma \pi^{d}\right)\left(\varepsilon^{-1} b^{-1} \pi^{-r / 2} a\right)^{2}\right) \\
= & \varepsilon \pi^{r} b^{2}\left(\left(1+\varepsilon^{-1} b^{-1} \pi^{-r / 2} a\right)^{2}\right.
\end{aligned}
$$

is in $\varepsilon\left(1+p^{e-[e / 2-r / 4]}\right) \dot{F}^{2}$.

If ord $a<r / 2$, ord $b=0$; then

$$
\begin{aligned}
& \operatorname{ord}\left(-2\left(1+a^{-1} b \pi^{r / 2}\right) a^{-1} b \pi^{r / 2}\right) \\
& \quad=e-\operatorname{ord} a+r / 2>e \geq e-[e / 2-r / 4]
\end{aligned}
$$


and

$$
\begin{aligned}
& \operatorname{ord}\left(-\sigma\left(a^{-1} b \pi^{r / 2}\right)^{2} \pi^{d}\right)=r+d-2 \text { ord } a \\
& \quad>d>e+[e / 2-r / 4] \geq e-[e / 2-r / 4]
\end{aligned}
$$

So

$Q(z)=a^{2}\left(\left(1+a^{-1} b \pi^{r / 2}\right)^{2}-2\left(1+a^{-1} b \pi^{r / 2}\right) a^{-1} b \pi^{r / 2}-\sigma\left(a^{-1} b \pi^{r / 2}\right)^{2} \pi^{d}\right)$

is in $\left(1+p^{e-[e / 2-r / 4]}\right) \dot{F}^{2}$. If ord $a=r / 2$, ord $b=0$, and

$$
\operatorname{ord}\left(\varepsilon+\left(b^{-1} a \pi^{-r / 2}\right)^{2}\right) \leq e-r / 2,
$$

note

$$
\operatorname{ord}\left(\sigma\left(a^{-1} b \pi^{r / 2}\right)^{2} \pi^{d}\right)=d>e+(e / 2-r / 4) \geq e>e-r / 2
$$

and

$\operatorname{ord}\left(2\left(1+a^{-1} b \pi^{r / 2}\right)\left(a^{-1} b \pi^{r / 2}\right)\right)=e+\operatorname{ord}\left(1+a^{-1} b \pi^{r / 2}\right) \geq e>e-r / 2$

Then

$$
\begin{aligned}
& \operatorname{ord}\left(1+a^{-1} b \pi^{r / 2}\right)^{2} \\
& \begin{aligned}
&=\operatorname{ord}\left(\left(1+a^{-1} b \pi^{r / 2}\right)^{2}-2\left(1+a^{-1} b \pi^{r / 2}\right)\left(a^{-1} b \pi^{r / 2}\right)\right. \\
&- \sigma\left(a^{-1} b \pi^{r / 2}\right)^{2} \pi^{d} \\
&\left.+2\left(1+a^{-1} b \pi^{r / 2}\right)\left(a^{-1} b \pi^{r / 2}\right)+\sigma\left(a^{-1} b \pi^{r / 2}\right)^{2} \pi^{d}\right)
\end{aligned} \\
& =\operatorname{ord}\left(\left(1+\varepsilon\left(a^{-1} b \pi^{r / 2}\right)^{2}\right)+2\left(1+a^{-1} b \pi^{r / 2}\right)\left(a^{-1} b \pi^{r / 2}\right)\right. \\
& \left.\quad+\sigma\left(a^{-1} b \pi^{r / 2}\right)^{2} \pi^{d}\right) \\
& =\operatorname{ord}\left(1+\varepsilon\left(a^{-1} b \pi^{r / 2}\right)^{2}\right) \leq e-r / 2 .
\end{aligned}
$$

So

$$
\begin{aligned}
& \operatorname{ord}\left(-2\left(1+a^{-1} b \pi^{r / 2}\right)^{-1}\left(a^{-1} b \pi^{r / 2}\right)\right) \\
& \quad=e-\operatorname{ord}\left(1+a^{-1} b \pi^{r / 2}\right) \geq e-[e / 2-r / 4]
\end{aligned}
$$

and

$$
\begin{aligned}
& \operatorname{ord}\left(-\sigma\left(a^{-1} b \pi^{r / 2}\right)^{2} \pi^{d}\left(1+a^{-1} b \pi^{r / 2}\right)^{-2}\right) \\
& \quad=d-2 \operatorname{ord}\left(1+a^{-1} b \pi^{r / 2}\right) \geq d-e+r / 2 \geq e-[e / 2-r / 4] .
\end{aligned}
$$

\section{Consider}

$$
\begin{aligned}
Q(z)=a^{2}\left(1+a^{-1} b \pi^{r / 2}\right)^{2}(1 & -2 a^{-1} b \pi^{r / 2}\left(1+a^{-1} b \pi^{r / 2}\right)^{-1} \\
& \left.-\sigma\left(a^{-1} b \pi^{r / 2}\right)^{2} \pi^{d}\left(1+a^{-1} b \pi^{r / 2}\right)^{-2}\right)
\end{aligned}
$$

is in $\left(1+p^{e-[e / 2-r / 4]}\right) \dot{F}^{2}$. Therefore

$$
\theta\left(O^{+}(L)\right) \subseteq\left(1+p^{e-[e / 2-r / 4]}\right) \dot{F}^{2} \cup \varepsilon\left(1+p^{e-[e / 2-r / 4]}\right) \dot{F}^{2} .
$$


Conversely, take an element $h=1+c \pi^{e-[e / 2-r / 4]}$ in $\left(1+p^{e-[e / 2-r / 4]}\right) \dot{F}^{2}$, and write $c=\lambda \pi^{k}$ with $\lambda \in U, k \geq 0$. If $0 \leq k \leq[e / 2-r / 4]$, there exists $\eta$ in $U$ such that

$$
\sigma \pi^{d-e-[e / 2-r / 4]+k} \eta^{2}+2 \pi^{-e} \eta+\lambda=0
$$

by Hensel's lemma. Put $z=\left(-1+\eta^{-1} \pi^{[e / 2-r / 4]-k}\right) \pi^{r / 2} x+y$ when $-1+\eta^{-1} \pi^{[e / 2-r / 4]-k}$ is in $U$, note

$$
\operatorname{ord}\left(-\sigma \pi^{d}\right)=d>e+[e / 2-r / 4]>2[e / 2-r / 4] \geq 2[e / 2-r / 4]-2 k
$$

and

$$
\operatorname{ord}\left(-2 \eta^{-1} \pi^{[e / 2-r / 4]-k}\right)=e+[e / 2-r / 4]-k>2[e / 2-r / 4]-2 k
$$

So

$$
\begin{aligned}
\operatorname{ord}( & \left.\left(-1+\eta^{-1} \pi^{[e / 2-r / 4]-k}\right)^{2}+\varepsilon\right) \\
& =\operatorname{ord}\left(1+\eta^{-2} \pi^{2[e / 2-r / 4]-2 k}-2 \eta^{-1} \pi^{[e / 2-r / 4]-k}-1-\sigma \pi^{d}\right) \\
& =\operatorname{ord}\left(\eta^{-2} \pi^{2[e / 2-r / 4]-2 k}-2 \eta^{-1} \pi^{[e / 2-r / 4]-k}-\sigma \pi^{d}\right) \\
& =\operatorname{ord}\left(\eta^{-2} \pi^{2[e / 2-r / 4]-2 k}\right) \\
& =2[e / 2-r / 4]-2 k \leq 2[e / 2-r / 4] \leq e-r / 2 .
\end{aligned}
$$

Thus, $\tau_{z}$ is in $O(L)$, and

$$
\begin{aligned}
Q(z)= & \pi^{r}\left(\left(-1+\eta^{-1} \pi^{[e / 2-r / 4]-k}\right)^{2}+\varepsilon\right) \\
= & \pi^{r}\left(\eta^{-2} \pi^{2[e / 2-r / 4]-2 k}-2 \eta^{-1} \pi^{[e / 2-r / 4]-k}-\sigma \pi^{d}\right) \\
= & \pi^{r+2[e / 2-r / 4]-2 k} \eta^{-2}\left(1-2 \eta \pi^{k-[e / 2-r / 4]}-\sigma \eta^{2} \pi^{d+2 k-[e / 2-r / 4]}\right) \\
= & \eta^{-2} \pi^{r+2[e / 2-r / 4]-2 k} \\
& \times\left(1+\pi^{k-[e / 2-r / 4]+e}\left(-2 \pi^{-e} \eta-\sigma \eta^{2} \pi^{d+k-[e / 2-r / 4]-e}\right)\right) \\
= & \eta^{-2} \pi^{r+2[e / 2-r / 4]-2 k}\left(1+\lambda \pi^{k-[e / 2-r / 4]+e}\right) \\
= & \eta^{-2} \pi^{r+2[e / 2-r / 4]-2 k}\left(1+c \pi^{e-[e / 2-r / 4]}\right) \\
= & \eta^{-2} \pi^{r+2[e / 2-r / 4]-2 k} h .
\end{aligned}
$$

If $k>[e / 2-r / 4]$, there exists $\eta$ in $U$ such that

$$
\begin{aligned}
\lambda \eta^{2} & +\left(2 \pi^{-e}+2 \lambda \pi^{k-[e / 2-r / 4]}\right) \eta+\lambda \pi^{2 k-2[e / 2-r / 4]} \\
& +2 \pi^{-e+k-[e / 2-r / 4]}+\sigma \pi^{d-e+k-[e / 2-r / 4]}=0
\end{aligned}
$$


by Hensel's lemma. Put $z=\eta \pi^{r / 2+[e / 2-r / 4]-k} x+y$, note $r / 2+$ $[e / 2-r / 4]-k<r / 2$, so $\tau_{z}$ is in $O(L)$, and

$$
\begin{aligned}
Q(z)= & \eta^{2} \pi^{r+2[e / 2-r / 4]-2 k}+\varepsilon \pi^{r} \\
= & \pi^{r+2[e / 2-r / 4]-2 k}\left(\eta^{2}-\pi^{2 k-2[e / 2-r / 4]}-\sigma \pi^{d-2[e / 2-r / 4]+2 k}\right) \\
= & \pi^{r+2[e / 2-r / 4]-2 k}\left(\left(\eta+\pi^{k-[e / 2-r / 4]}\right)^{2}+\pi^{e+k-[e / 2-r / 4]}\right. \\
& \left.\quad \times\left(-2 \eta \pi^{-e} 2 \pi^{-e+k-[e / 2-r / 4]}-\sigma \pi^{d-e+k-[e / 2-r / 4]}\right)\right) \\
= & \pi^{r+2[e / 2-r / 4]-2 k}\left(\left(\eta+\pi^{k-[e / 2-r / 4]}\right)^{2}+\pi^{e+k-[e / 2-r / 4]}\right. \\
& \left.\times\left(\lambda \eta^{2}+2 \lambda \pi^{k-[e / 2-r / 4]} \eta+\lambda \pi^{2 k-2[e / 2-r / 4]}\right)\right) . \\
= & \pi^{r+2[e / 2-r / 4]-2 k}\left(\eta+\pi^{k-[e / 2-r / 4]}\right)^{2}\left(1+\lambda \pi^{k} \pi^{e-[e / 2-r / 4]}\right) \\
= & \pi^{r+2[e / 2-r / 4]-2 k}\left(\eta+\pi^{k-[e / 2-r / 4]}\right)^{2} h .
\end{aligned}
$$

Therefore

$$
\theta\left(O^{+}(L)\right)=\left(1+p^{e-[e / 2-r / 4]}\right) \dot{F}^{2} \cup \varepsilon\left(1+p^{e-[e / 2-r / 4]}\right) \dot{F}^{2}
$$

Remark 1. Suppose two units $\varepsilon, \eta$ with $D(\varepsilon)=p^{s}$ and $D(\eta)=$ $p^{t}$, where $s, t$ are odd integers, and $s+t>2 e$; then the Hilbert symbol $(\varepsilon, \eta)_{p}=1$. This fact can be proved by an argument similar to that of the case (iii) of Proposition 2.2 or [5, Lemma 4]. On the other hand, consider a unit $\varepsilon$ with $D(\varepsilon)=p^{s}$ where $s$ is an odd integer. For any positive odd integer $t$, if $s=t \leq 2 e$, then there exists a unit $\eta$ with $D(\eta)=p^{t}$ and the Hilbert symbol $(\varepsilon, \eta)_{p}=-1$. This result can be obtained from [5, Lemma 3].

Remark 2. Suppose $E$ is a finite extension of $F$ and $\bar{L}$ is the lifting of $L$ to $E$, then we can check $N_{E / F}\left(\theta\left(O^{+}(\bar{L})\right)\right) \subseteq \theta\left(O^{+}(L)\right)$ by the results obtained in [5], [10] and this section. These can give an alternative proof about spinor genus extension of binary lattices (see [2], [4]).

3. Main results. In this section let $L$ be a lattice with $\operatorname{dim} F L \geq 3$ and Jordan splitting $L=\vartheta x_{1} \perp \vartheta x_{2} \perp \cdots \perp \vartheta x_{n}$ where $Q\left(x_{1}\right)=1$, $Q\left(x_{i}\right)=\varepsilon_{i} \pi^{r_{i}}, r_{i} \in Z, \varepsilon_{i} \in U, i=2,3, \ldots, n$; and $0<r_{i}<r_{i+1}$, $i=2,3, \ldots, n-1$. We assume $r_{1}=0, \varepsilon_{1}=1$. First we generalize [3, Th. 2.2] to an arbitrary dyadic local field.

THEOREM 3.1. Suppose there is at least one $k$ with $1 \leq k \leq n-1$ for which $r_{k+1}-r_{k} \leq 2 e+1$ and $r_{k+1}-r_{k}$ is odd. Then if $0<r_{s}-r_{t} \leq 4 e$ and $r_{s}-r_{t}$ is even for any $s, t=1, \ldots, n$, we have $\theta\left(O^{+}(L)\right)=\dot{F}$. 
Proof. Put $L_{k+1, k}=\vartheta x_{k} \perp \vartheta x_{k+1}$. When $r_{k+1}-r_{k}=2 e+1$, then

$$
\begin{aligned}
\theta\left(O^{+}(L)\right) & =\left((1+p) \dot{F}^{2} \cup \varepsilon \pi(1+p) \dot{F}^{2}\right) \cap Q\left(\left[1, \varepsilon_{k} \dot{\varepsilon}_{k+1} \pi\right]\right) \\
& =\left(U \dot{F}^{2} \cup \pi U \dot{F}^{2}\right) \cap Q\left(\left[1, \varepsilon_{k} \dot{\varepsilon}_{k+1} \pi\right]\right)=Q\left(\left[1, \varepsilon_{k} \dot{\varepsilon}_{k+1} \pi\right]\right)
\end{aligned}
$$

by case (i) of Proposition 2.2. When $r_{k+1}-r_{k}<2 e$, we also obtain $\theta\left(O^{+}\left(L_{k+1, k}\right)\right)=Q\left(\left[1, \varepsilon_{k} \dot{\varepsilon}_{k+1} \pi\right]\right)$ by case (i) of Proposition 2.3. Note $Q\left(\left[1, \varepsilon_{k} \dot{\varepsilon}_{k+1} \pi\right]\right)$ is a subgroup of $\dot{F}$ with index 2 which does not contain $\Delta$.

Put $L_{s, t}=\vartheta x_{s} \perp \vartheta x_{t}$. By cases (ii), (iii) of Proposition 2.2 and Proposition 2.3, we check $\Delta$ in $\theta\left(O^{+}\left(L_{s, t}\right)\right)$. Hence $\theta\left(O^{+}(L)\right)=\dot{F}$.

Let $S(L)$ be the group generated by symmetries in $O(L)$ (see [7]). We need the following lemma.

LEMMA 3.2. If $r_{i+1}-r_{i} \geq e$, for all $i=1,2, \ldots, n-1$, with at most one exception, then $O(L)=S(L)$.

Proof. First we assume $r_{2} \geq e$, otherwise we scale the dual lattice of $L$ by $\varepsilon_{n} \pi^{r_{n}}$ and obtain the lattice $L^{\prime}$ which satisfies this assumption, and we know $O(L)=O\left(L^{\prime}\right)$.

Take $\sigma$ in $O(L)$. Suppose $\sigma x_{1}=\sum_{i=1}^{n} a_{i} x_{i}, a_{i} \in \vartheta$. So

$$
1=Q\left(\sigma x_{1}\right)=Q\left(\sum_{i=1}^{n} a_{i} x_{i}\right)=\sum_{i=1}^{n} \varepsilon_{i} \pi^{r_{\iota}} a_{i}^{2} .
$$

If $\operatorname{ord}\left(a_{1}-1\right) \leq e$, then

$$
\text { ord } Q\left(\sigma x_{1}-x_{1}\right)=\text { ord }\left(\left(a_{1}-1\right)^{2}+\sum_{i=2}^{n} a_{i}^{2} \varepsilon_{i} \pi^{r_{\imath}}\right)=\text { ord } 2\left(a_{1}-1\right)
$$

and

$$
2 B\left(\sigma x_{1}-x_{1}, L\right) \vartheta=2\left(a_{1}-1\right) \vartheta=Q\left(\sigma x_{1}-x_{1}\right) \vartheta
$$

Thus, $\tau_{\sigma x_{1}-x_{1}}$ is in $O(L)$.

If $\operatorname{ord}\left(a_{1}-1\right)>e$, then $\operatorname{ord}\left(a_{1}+1\right)=\operatorname{ord}\left(2+\left(a_{1}-1\right)\right)=e$, and

$$
\text { ord } Q\left(\sigma x_{1}+x_{1}\right)=\text { ord }\left(\left(a_{1}+1\right)^{2}+\sum_{i=2}^{n} a_{i}^{2} \varepsilon_{i} \pi^{r_{\imath}}\right)=\text { ord } 2\left(a_{1}+1\right)
$$

and

$$
2 B\left(\sigma x_{1}+x_{1}, L\right) \vartheta=2\left(a_{1}+1\right) \vartheta=Q\left(\sigma x_{1}+x_{1}\right) \vartheta .
$$

Thus, $\tau_{\sigma x_{1}+x_{1}}$ is in $O(L)$. 
Since $\tau_{\sigma x_{1}-x_{1}} \sigma$ and $\tau_{x_{1}} \tau_{\sigma x_{1}+x_{1}} \sigma$ can be regarded as elements in $O(K)$ where $K=\vartheta x_{2} \perp \cdots \perp \vartheta x_{n}$, by induction on $n$, we obtain $O(L)=S(L)$.

Now we deduce the Kneser-type result about spinor norms.

THEOREM 3.3. If $r_{i+1}-r_{i}>4 e$, for all $i=1,2, \ldots, n-1$; then

$$
\theta\left(O^{+}(L)\right)=\bigcup_{k \text { even }} \varepsilon_{i_{1}} \cdots \varepsilon_{i_{k}} \pi^{r_{i_{1}}+\cdots+r_{i_{k}} \dot{F}^{2}}
$$

Proof. Take a symmetry $\tau_{z}$ in $O(L)$, where $z$ is a maximal anisotropic vector in $L$. Then $2 B(z, L) \vartheta \subseteq Q(z) \vartheta$. Write $z=\sum_{i=1}^{n} a_{i} x_{i}$ with $a_{i} \in \vartheta$, so

$$
\text { ord } Q(z)=\text { ord }\left(\sum_{i=1}^{n} a_{i}^{2} \varepsilon_{i} \pi^{r_{t}}\right) \leq \min _{1 \leq i \leq n}\left\{r_{i}+\operatorname{ord} a_{i}\right\}+e
$$

Choose $k$ so that $\operatorname{ord}\left(a_{k}^{2} \varepsilon_{k} \pi^{r_{k}}\right)=\min _{1 \leq i \leq n}\left\{\operatorname{ord}\left(a_{i}^{2} \varepsilon_{i} \pi^{r_{\imath}}\right)\right\}$. Then

$$
2 \text { ord } a_{k}+r_{k} \leq \text { ord } Q(z) \leq \min _{1 \leq i \leq n}\left\{r_{i}+\text { ord } a_{i}\right\}+e \leq r_{j}+\text { ord } a_{j}+e
$$

for $j=1,2, \ldots, n$. When we take $j=k$, ord $a_{k} \leq e$ is obtained.

When $j<k$, we have

$$
\begin{aligned}
& \operatorname{ord}\left(\left(\varepsilon_{j} \varepsilon_{k}^{-1}\right)\left(a_{j} a_{k}^{-1}\right)^{2} \pi^{r_{j}-r_{k}}\right)=2 \text { ord } a_{j}-2 \text { ord } a_{k}+r_{j}-r_{k} \\
& \quad=2\left(\text { ord } a_{j}-2 \text { ord } a_{k}-r_{k}+r_{j}+e\right)+2 \text { ord } a_{k}+\left(r_{k}-r_{j}\right)-2 e \\
& \quad \geq\left(r_{k}-r_{j}\right)-2 e>2 e .
\end{aligned}
$$

When $j>k$, consider

$$
\begin{aligned}
& \operatorname{ord}\left(\left(\varepsilon_{j} \varepsilon_{k}^{-1}\right)\left(a_{j} a_{k}^{-1}\right)^{2} \pi^{r_{j}-r_{k}}\right)=2 \text { ord } a_{j}-2 \text { ord } a_{k}+r_{j}-r_{k} \\
& \quad \geq r_{j}-r_{k}-2 \text { ord } a_{k} \geq r_{j}-r_{k}-2 e>2 e .
\end{aligned}
$$

So

$$
\begin{aligned}
Q(z)=\varepsilon_{k} \pi^{r_{k}} a_{k}^{2}\left(1+\sum_{i=1}^{k-1}\left(\varepsilon_{k}^{-1} \varepsilon_{i}\right)\left(a_{k}^{-1} a_{i}\right)^{2} \pi^{r_{\imath}-r_{k}}\right. & \\
& \left.+\sum_{i=k+1}^{n}\left(\varepsilon_{k}^{-1} \varepsilon_{i}\right)\left(a_{k}^{-1} a_{i}\right)^{2} \pi^{r_{\imath}-r_{k}}\right)
\end{aligned}
$$

is in $\varepsilon_{k} \pi^{r} \dot{F}^{2}$ by the Local Square Theorem. By Lemma 3.2, we obtain $\theta\left(O^{+}(L)\right)=\bigcup_{k \text { even }} \varepsilon_{i_{1}} \cdots \varepsilon_{i_{k}} \pi^{r_{i_{1}}+\cdots+r_{i_{k}} \dot{F}^{2}}$. 
4. Application. In this section we will show that the restriction in [1], that $F$ be a ramified quadratic extension of $Q_{2}$, is unnecessary. In fact we have the following theorem.

THEOREM 4.1. Suppose $\vartheta$ is the ring of integers of dyadic local field $F$ with $e=$ ord $2=2$. Let $L$ be a regular $\vartheta$-lattice with $s L \subseteq \vartheta$ and rank $L \geq 3$. If $\operatorname{ord}(d L) \leq 3$ then $\theta\left(O^{+}(L)\right) \supseteq U \dot{F}^{2}$.

Proof. Let $L=L_{1} \perp \cdots \perp L_{t}$ be a Jordan splitting of $L$. We can assume $t \geq 2$ and $\operatorname{dim} F L_{i} \leq 2, i=1, \ldots, t$, by [8, 93:20], and $2 s L_{i} \subset n L_{i}$ by [5, Lemma 1], $i=1, \ldots, t$. Since $\operatorname{ord}(d L) \leq 3$, $t \leq 3$.

(1) $L=L_{1} \perp L_{2}$ with rank $L_{1}=\operatorname{rank} L_{2}=2$, so $L_{1}$ is unimodular and $s L_{2}=p$. By $[8,93: 17]$ we may write $L_{i}=\vartheta x_{i}+\vartheta y_{i}$ with $Q\left(x_{i}\right)=a_{i}, Q\left(y_{i}\right)=-\delta_{i} a_{i}^{-1}, B\left(x_{i}, y_{i}\right)=\pi^{i-1}$, and $a_{i} \vartheta=n L_{i}$, $\delta_{i} \vartheta=D\left(-d L_{i}\right), i=1,2$.

If $n L_{1}=p s L_{1}=p$, we only need to consider $D\left(-d L_{1}\right)=p^{3}$ by [5, Prop. C], and $\theta\left(O^{+}\left(L_{1}\right)\right)$ contains $\Delta$ in this case by [5, Prop. B].

Suppose $n L_{2}=p s L_{2}=p^{2}$. Put $K=\vartheta x_{1} \perp \vartheta x_{2}$. Consider any maximal vector of $K$, say $z$, which is then also a maximal anisotropic vector of $L$. We can check that $2 B(z, L) \vartheta \subseteq Q(z) \vartheta$, so $\tau_{z}$ is in $O(L)$, and $\theta\left(O^{+}(L)\right) \supseteq Q\left(\left[1, \dot{a}_{1} a_{2}\right]\right) \dot{F}^{2}$ which is a subgroup of $\dot{F}$ with index 2 and does not contain $\Delta$. Therefore $\theta\left(O^{+}(L)\right)=\dot{F}$.

Suppose $n L_{2}=s L_{2}=p$. Put $K=\vartheta y_{1} \perp \vartheta x_{2}$, by taking the same argument as above, we obtain $\theta\left(O^{+}(L)\right)=\dot{F}$ again.

If $n L_{1}=s L_{1}=\vartheta$, then $\theta\left(O^{+}\left(L_{1}\right)\right)$ contains $\Delta$ by the results obtained in [5] and [10].

Suppose $n L_{2}=p s L_{2}=p^{2}$. By [5, Prop. C], we only need to treat the case of $D\left(-d L_{2}\right)=p^{5}$. Put $K=\vartheta x_{1} \perp \vartheta y_{2}$. Note any maximal vector of $K$, say $z$, which is also a maximal anisotropic vector of $L$, satisfies $2 B(z, L) \vartheta \subseteq Q(z) \vartheta$. So $\tau_{z}$ is in $O(L), \theta\left(O^{+}(L)\right) \supseteq$ $Q\left(\left[1, \dot{a}_{1} b_{2}\right]\right)$ which does not contain $\Delta$. Therefore $\theta\left(O^{+}(L)\right)=\dot{F}$. $\dot{F}$.

Suppose $n L_{2}=s L_{2}=p$. Put $K=\vartheta x_{1} \perp \vartheta x_{2}$. Then $\theta\left(O^{+}(L)\right)=$

(2) $L=L_{1} \perp L_{2}$ with $\operatorname{rank} L_{1}=2, \operatorname{rank} L_{2}=1$, so $L_{1}$ is unimodular.

Write $L_{1}=\vartheta x_{1}+\vartheta y_{1}$ with $Q\left(x_{1}\right)=a_{1}, Q\left(y_{1}\right)=-\delta_{1} a_{1}^{-1}, B\left(x_{1}, y_{1}\right)$ $=1$, and $n L_{1}=a_{1} \vartheta, D\left(-d L_{1}\right)=\delta_{1} \vartheta$ by [8,93:17]. Let $L_{2}=\vartheta x_{2}$ with $Q\left(x_{2}\right)=a_{2} \in p$. If $n L_{1}=p s L_{1}=p$, by [5, Prop. C], we only need to consider $D\left(-d L_{1}\right)=p^{3}$, and $\theta\left(O^{+}\left(L_{1}\right)\right)$ contains $\Delta$ in this case by [5, Prop. B]. 
When $Q\left(x_{2}\right) \vartheta=a_{2} \vartheta=p$, put $K=\vartheta y_{1} \perp \vartheta x_{2}$. Then $\theta\left(O^{+}(L)\right)=$ $\dot{F}$. When $Q\left(x_{2}\right) \vartheta=p^{2}$, put $K=\vartheta x_{1} \perp \vartheta x_{2}$. Then $\theta\left(O^{+}(L)\right)=\dot{F}$. When $Q\left(x_{2}\right) \vartheta=p^{3}$, put $K=\vartheta y_{1} \perp \vartheta x_{2}$. Then $\theta\left(O^{+}(L)\right)=\dot{F}$.

If $n L_{1}=s L_{1}=\vartheta$, [5, Prop. C], we only need to consider $\delta_{1} \vartheta=p$ or $p^{3}$, and we know that $\theta\left(O^{+}\left(L_{1}\right)\right)$ contains $\Delta$ in these two cases by [5, Prop. B,E]. When $Q\left(x_{2}\right) \vartheta=p$, put $K=\vartheta x_{1} \perp \vartheta x_{2}$. Then $\theta\left(O^{+}(L)\right)=\dot{F}$. When $Q\left(x_{2}\right) \vartheta=p^{3}$, put $K=\vartheta x_{1} \perp \vartheta x_{2}$. Then $\theta\left(O^{+}(L)\right)=\dot{F}$. When $Q\left(x_{2}\right) \vartheta=p^{2}$, and $\delta_{1} \vartheta=p$, put $K=\vartheta y_{1} \perp$ $\vartheta x_{2}$. Then $\theta\left(O^{+}(L)\right)=\dot{F}$. When $Q\left(x_{2}\right) \vartheta=a_{2} \vartheta=p^{2}$, and $\delta_{1} \vartheta=p^{3}$; we know $\left(1+p^{2}\right) \dot{F}^{2} \subseteq \theta\left(O^{+}\left(L_{1}\right)\right)$ by [5, Prop. E]. Write $a_{2}=\sigma \pi^{2}$ with $\sigma \in U,-a_{1}^{-1} \delta_{1}=\eta \pi^{3}$ with $\eta \in U$. Take an element $1+\lambda \pi$ in $1+p$ with $\lambda \in U$.

Suppose $\lambda \sigma \eta^{-1}=\xi^{2}+\varepsilon \pi^{d}$ with $\xi, \varepsilon \in U$ and $d \geq 4$; then there exists $t$ in $\vartheta$ such that $a_{1} t^{2}+2 \pi^{-2} \xi t-\eta \varepsilon \pi^{d-1}=0$ by Hensel's lemma. Put $z=\pi^{2} t x_{1}+\xi y_{1}+x_{2}$, which is a maximal vector of $L$, and

$$
\begin{aligned}
Q(z) & =\pi^{4} t^{2} a_{1}+2 \pi^{2} \xi t+\xi^{2} \eta \pi^{3}+\sigma \pi^{2} \\
& =\pi^{4} \eta \varepsilon \pi^{d-1}+\xi^{2} \eta \pi^{3}+\sigma \pi^{2}=\eta \pi^{3}\left(\xi^{2}+\varepsilon \pi^{d}\right)+\sigma \pi^{2} \\
& =\lambda \sigma \pi^{3}+\sigma \pi^{2}=\sigma \pi^{2}(1+\lambda \pi) .
\end{aligned}
$$

So $2 B(z, L) \vartheta \subseteq Q(z) \vartheta$, and $\tau_{z}$ is in $O(L)$. Then $(1+\lambda \pi)$ is in $\theta\left(O^{+}(L)\right)$. Suppose $\lambda \sigma \eta^{-1}=\xi^{2}+\varepsilon \pi^{d}$ with $\xi, \varepsilon \in U$ and $1 \leq d \leq 3$, and $d$ is odd. Put $\sigma^{-1} \eta \varepsilon=\omega^{2}+\delta \pi^{h}$ with $\omega, \delta \in U$. Then there exists $t$ in $\vartheta$ such that $\sigma^{-1} a_{1} t^{2}+\left(2 \pi^{-2}\right) \sigma^{-1} \xi t-\delta \pi^{d+h-1}+$ $\left(2 \pi^{-2}\right) \omega \pi^{(d+1) / 2}=0$ by Hensel's lemma. Put $z=\pi^{2} t x_{1}+\xi y_{1}+$ $\left(1+\omega \pi^{(d+1) / 2}\right) x_{2}$ which is a maximal vector of $L$, and

$$
\begin{aligned}
Q(z)= & \tau^{4} t^{2} a_{1}+2 \pi^{2} t \xi+\xi^{2} \pi^{3} \eta+\left(1+\omega \pi^{(d+1) / 2}\right)^{2} \sigma \pi^{2} \\
= & \sigma \pi^{2}\left(\pi^{2}\left(\sigma^{-1} a_{1} t^{2}+2 \pi^{-2} \sigma^{-1} \xi t\right)\right. \\
& \left.\quad+\sigma^{-1} \xi^{2} \pi \eta+1+2 \omega \pi^{(d+1) / 2}+\omega^{2} \pi^{d+1}\right) \\
= & \quad+2 \omega \pi^{2}\left(\delta \pi^{d+h+1}-2 \omega \pi^{(d+1) / 2}+\sigma^{-1} \xi^{2} \pi \eta+1\right. \\
& \\
= & \sigma \pi^{2}\left(1+\sigma^{-1} \xi^{2} \eta \pi+\pi^{d+1}\left(\omega^{2}+\delta \pi^{h}\right)\right) \\
= & \sigma \pi^{2}\left(1+\sigma^{-1} \xi^{2} \eta \pi+\sigma^{-1} \eta \varepsilon \pi^{d+1}\right) \\
= & \sigma \pi^{2}\left(1+\sigma^{-1} \eta \pi\left(\xi^{2}+\varepsilon \pi^{d}\right)\right) \\
= & \sigma \pi^{2}\left(1+\sigma^{-1} \eta \pi \lambda \sigma \eta^{-1}\right)=\sigma \pi^{2}(1+\lambda \pi) .
\end{aligned}
$$

So $2 B(z, L) \subseteq Q(z) \vartheta$, and $\tau_{z}$ is in $O(L)$, then $(1+\lambda \pi)$ is in $\theta\left(O^{+}(L)\right)$. Therefore, $\theta\left(O^{+}(L)\right) \supseteq(1+p) \dot{F}^{2}=U \dot{F}^{2}$. 
(3) $L=L_{1} \perp L_{2}$ with rank $L_{1}=1$, rank $L_{2}=2$. We scale the dual lattice of $L$ by $\pi$ and reduce to case (2).

(4) $L=L_{1} \perp L_{2} \perp L_{3}$ with $\operatorname{rank} L_{1}=\operatorname{rank} L_{2}=\operatorname{rank} L_{3}=1$. Let $L_{i}=\vartheta x_{i}, i=1,2,3$; so $Q\left(x_{i}\right) \vartheta=p^{i-1}, 1 \leq i \leq 3$. Therefore $\theta\left(O^{+}(L)\right)=\dot{F}$ by Theorem 3.1 .

(5) $L=L_{1} \perp L_{2} \perp L_{3}$ with rank $L_{1}=2$, rank $L_{2}=\operatorname{rank} L_{3}=1$. So $L_{1}$ is unimodular, and $L_{i}=\vartheta x_{i}$ with $Q\left(x_{i}\right) \vartheta=p^{i-1}, i=2.3$. Since $\Delta$ is in $\theta\left(O^{+}\left(L_{1}\right)\right)$ by the results obtained in [5] and [10], put $K=\vartheta x_{2} \perp \vartheta x_{3}$, obtain $\theta\left(O^{+}(L)\right)=\dot{F}$.

LEMMA 4.2. Suppose $\vartheta$ is the ring of integers of a dyadic local field with $e=$ ord $2=2$. Let $L$ be a regular $\vartheta$-lattice with $s L \subseteq \vartheta$ and rank $L \geq 4$. If $\operatorname{ord}(d L) \leq 7$, then $\theta\left(O^{+}(L)\right) \supseteq U \dot{F}^{2}$.

Proof. Using the above theorem, considering components and dual lattices when necessary, there remain two cases to be treated.

(1) $L=L_{1} \perp L_{2}$ where $L_{1}$ is binary unimodular, $L_{2}$ is binary $p^{2}$-modular. Write $L_{1}=\vartheta x_{1} \perp \vartheta y_{1}$ with $Q\left(x_{1}\right)=a_{1}, B\left(x_{1}, y_{1}\right)=$ $1, Q\left(y_{1}\right)=-\delta_{1} a^{-1}$, and $n L_{1}=a_{1} \vartheta, D\left(-d L_{1}\right)=\delta_{1} \vartheta$; and $L_{2}=$ $\vartheta x_{2}+\vartheta y_{2}$ with $Q\left(x_{2}\right)=a_{2}, B\left(x_{2}, y_{2}\right)=\pi^{2}, Q\left(y_{2}\right)=-\delta_{2} a_{2}^{-1}$, and $n L_{2}=a_{2} \vartheta . D\left(-d L_{2}\right)=\delta_{2} \vartheta$ by $[8,93: 17]$.

We know that $L_{i}$ has an orthogonal base if and only if $n L_{i}=$ $s L_{i} \quad(i=1,2)$. Using the above theorem, considering components and dual lattices when necessary, we assume $n L_{1}=p\left(s L_{1}\right)=p$, and $n L_{2}=p\left(s L_{2}\right)=p^{3}$. By [5, Prop. C], we only need to consider $D\left(-d L_{1}\right)=p^{3}$, and $\Delta$ is in $\theta\left(O^{+}\left(L_{1}\right)\right)$ by [5, Prop. B] in this case. Put $K=\vartheta y_{1} \perp \vartheta x_{2}$. Then $\theta\left(O^{+}(L)\right)=\dot{F}$.

(2) $L=L_{1} \perp L_{2}$ where $L_{1}$ is binary unimodular and $L_{2}$ is binary $p^{3}$-modular. We know $L_{i}$ has an orthogonal base if and only if $n L_{i}=$ $s L_{i} \quad(i=1,2)$. Using the above theorem, considering components and dual lattices when necessary, we assume $n L_{1}=p\left(s L_{1}\right)=p$ and $n L_{2}=p s L_{2}=p^{4}$. Write $L_{1}=\vartheta x_{1}+\vartheta y_{1}$ with $Q\left(x_{1}\right) \vartheta=n L_{1}=p$, and $L_{2}=\vartheta x_{2}+\vartheta y_{2}$ with $Q\left(x_{2}\right) \vartheta=n L_{2}=p^{4}$, note $\Delta$ is in $\theta\left(O^{+}\left(L_{1}\right)\right)$ by [5] and [10], put $K=\vartheta x_{1} \perp \vartheta x_{2}$, then $\theta\left(O^{+}(L)\right)=\dot{F}$.

By using Theorem 4.1, Lemma 4.2 and Theorem 3.1, we can also prove [1, Lemma 3.4] and [1, Theorem 3.5] when $\vartheta$ is the ring of integers of a dyadic local field with $e=$ ord $2=2$. Finally we consider an example.

EXAMPLE 4.3. The bound ord $(d L) \leq 7$ given in Lemma 4.2 cannot be unconditionally improved. Consider the lattice $L=L_{1} \perp L_{2}$ over 
the ring of integers of a ramified quadratic extension of $Q_{2}$, where $L_{1}=\vartheta x_{1}+\vartheta y_{1}$ with $Q\left(x_{1}\right)=1, Q\left(y_{1}\right)=\pi^{3}, B\left(x_{1}, y_{1}\right)=1$, and $L_{2}=\vartheta x_{2}+\vartheta y_{2}$ with $Q\left(x_{2}\right)=\pi^{4}, Q\left(y_{2}\right)=\pi^{7}, B\left(x_{2}, y_{2}\right)=\pi^{4}$. We claim $\theta\left(O^{+}(L)\right)=\left(1+p^{2}\right) \dot{F}^{2} \subset U \dot{F}^{2}$.

First we prove that $O(L)$ is generated by symmetries of $L$. Note $L_{i}$ has an orthogonal base $(i=1,2)$, and write $L_{1}=\vartheta z \perp \vartheta w$. Put $K=\vartheta w \perp L_{2}$. Then $O(K)$ is generated by symmetries of $K$ by the proof of Lemma 3.2. Let $\sigma \in O(L)$, and $\sigma z=a z+b w+u$ with $u \in L_{2}$, so $Q(z)=a^{2} Q(z)+b^{2} Q(w)+Q(u)$ and $Q(\sigma z-z)=$ $2(1-a) Q(z), Q(\sigma z+z)=2(1+a) Q(z)$.

If $\operatorname{ord}(1-a)=0$, then $2 B(\sigma z-z, L)=Q(\sigma z-z) \vartheta$, and $\tau_{\sigma z-z} \in$ $O(L)$. If $\operatorname{ord}(1-a)=1$, then $b^{2} Q(w)=(1-a)(1+a) Q(z)-Q(u) \in p$, and ord $b \geq 1$. So $2 B(\sigma z-z, L)=Q(\sigma z-z) \vartheta$, and $\tau_{\sigma z-z} \in O(L)$.

If $\operatorname{ord}(1-a)=2$, then $\operatorname{ord}(1+a)=\operatorname{ord}(2-(1-a))>2$, and $b^{2} Q(w)=(1+a)(1-a) Q(z)-Q(u) \in p^{4}$, so ord $b \geq 2$, and $2 B(\sigma z-z, L)=Q(\sigma z-z) \vartheta$. Thus $\tau_{\sigma z-z} \in O(L)$.

If $\operatorname{ord}(1-a)>2$, then $\operatorname{ord}(1+a)=\operatorname{ord}(2-(1-a))=2$, and $b^{2} Q(w)=(1+a)(1-a) Q(z)-Q(u) \in p^{4}$, so ord $b \geq 2$, and $2 B(\sigma z+z, L) \subseteq Q(\sigma z+z) \vartheta$.Thus $\tau_{\sigma z+z} \in O(L)$.

Note that $\tau_{\sigma z-z} \sigma$ and $\tau_{z} \tau_{\sigma z+z} \sigma$ can be regarded as elements in $O(K)$ which is generated by symmetries of $K$. Therefore $O(L)$ is generated by symmetries of $L$.

Now we calculate $\theta\left(O^{+}(L)\right)$. Let $\tau_{t} \in O(L)$ where $t$ is a maximal anisotropic vector of $L$, write $t=c x_{1}+d y_{1}+f x_{2}+g y_{2}$ with $c, d, f, g \in \vartheta$.

When ord $c \leq 1$, then $2 c^{-1} d+c^{-2} d^{2} \pi^{3}$ is always in $p^{2}$ whenever $c$ and $d$ are units or not. So

$$
Q(t)=c^{2}\left(1+\left(2 c^{-1} d+c^{-2} d^{2} \pi^{3}\right)+c^{-2} \pi^{4}\left(f^{2}+2 f g+g^{2} \pi^{3}\right)\right)
$$

is in $\left(1+p^{2}\right) \dot{F}^{2}$.

When $\operatorname{ord}(d)=0$, and $\operatorname{ord}(c)>1$; then ord $Q(t)=3$, and $2 B(t, L)=p^{2} \supset Q(t) \vartheta$, contradicting the assumption that $\tau_{t}$ is in $O(L)$.

When $\operatorname{ord}(f)=0, \operatorname{ord}(d)>0$, and $\operatorname{ord}(c)>1$; then $\operatorname{ord}(c) \geq 2$, and the inclusion $2 B(t, L) \subseteq Q(t) \vartheta$ forces $\operatorname{ord}(d) \geq 2$.

Suppose $\operatorname{ord}(c)=2$. Then $\operatorname{ord}\left(c^{2}+f^{2} \pi^{4}\right) \geq 5$. So

$$
\text { ord } Q(t)=\operatorname{ord}\left(\left(c^{2}+f^{2} \pi^{4}\right)+2 c d+d^{2} \pi^{3}+2 \pi^{4} f g+g^{2} \pi^{7}\right) \geq 5
$$

and $2 B(t, L)=p^{4} \supset Q(t) \vartheta$, a contradiction. 
Therefore $\operatorname{ord}(c) \geq 3$, and

$$
\begin{aligned}
Q(t)=f^{2} \pi^{4}\left(1+f^{-2} c^{2} \pi^{-4}+2 f^{-2} c d \pi^{-4}\right. & +d^{2} f^{-2} \pi^{-1} \\
& \left.+2 f^{-1} g+f^{-2} g^{2} \pi^{3}\right)
\end{aligned}
$$

is in $\left(1+p^{2}\right) \dot{F}^{2}$.

When $\operatorname{ord}(g)=0, \operatorname{ord}(f)>0, \operatorname{ord}(d)>0$, and $\operatorname{ord}(c)>1$; then $\operatorname{ord}(c) \geq 2$, and $\operatorname{ord}(d) \geq 2$.

Suppose $\operatorname{ord}(c)=2$. Then

$$
Q(t)=c^{2}\left(1+2 c^{-1} d+\left(c^{-1} d\right)^{2} \pi^{3}+c^{-2} \pi^{4}\left(f^{2}+2 f g+g^{2} \pi^{3}\right)\right)
$$

is in $\left(1+p^{2}\right) \dot{F}^{2}$.

Suppose $\operatorname{ord}(c) \geq 3$. Then $\operatorname{ord}(c) \geq 4$ and $\operatorname{ord}(d) \geq 4$.

If $\operatorname{ord}(f)=1$, then $2 f^{-1} g+\left(f^{-1} g\right)^{2} \pi^{3} \in p^{2}$, and

$$
\begin{array}{r}
Q(t)=f^{2} \pi^{4}\left(1+\left(f^{-1} c \pi^{-2}\right)^{2}+2 c d f^{-2} \pi^{-4}+d^{2} f^{-2} \pi^{-1}\right. \\
\left.+\left(2 f^{-1} g+\left(f^{-1} g\right)^{2} \pi^{3}\right)\right)
\end{array}
$$

is in $\left(1+p^{2}\right) \dot{F}^{2}$.

If $\operatorname{ord}(f) \geq 2$, then ord $Q(t)=7$, and $2 B(t, L)=p^{6} \supset Q(t) \vartheta$. But $\tau_{t}$ is in $O(L)$ and $t$ is a maximal vector, a contradiction. In any case, we have $\theta\left(O^{+}(L)\right) \subseteq\left(1+p^{2}\right) \dot{F}^{2}$. On the other hand, $\theta\left(O^{+}(L)\right) \supseteq \theta\left(O^{+}\left(L_{1}\right)\right) \supseteq\left(1+p^{2}\right) \dot{F}^{2}$ by [5, Prop. E]. Thus, we conclude $\theta\left(O^{+}(L)\right)=\left(1+p^{2}\right) \dot{F}^{2}$.

Acknowledgment. I wish to thank Professor $\mathrm{Zhu} \mathrm{Fu-zu} \mathrm{for} \mathrm{intro-}$ ducing me to the theory of quadratic forms and giving me much help in this field.

\section{REFERENCES}

[1] W. R. Bon Durant, Spinor norms of rotations of local integral quadratic forms, J. Number Theory, 33 (1989), 83-94.

[2] A. G. Earnest and D. R. Estes, Glass groups in the genus and spinor genus of binary quadratic lattices, Proc. London Math. Soc., 40 (1980), 40-52.

[3] A. G. Earnest and J. S. Hsia, Spinor norms of local integral rotations, II, Pacific J. Math., 61, No. 1 (1975), 71-86; errata 115 (1984), 493-494.

[4] _ Spinor genera under field extensions II: 2 unramified in the bottom field, Amer. J. Math., 100 (1978), 523-538.

[5] J. S. Hsia, Spinor norms of local integeral rotations, I, Pacific J. Math., 57 (1975), 199-206.

[6] M. Kneser, Klassenzahlen indefiniter quadratischer Formen in drei oder mehr Veranderlichen., Arch. Math., 7 (1956), 323-332.

[7] O. T. O'Meara and Barth Pollak, Generation of local integral orthogonal groups, II, Math. Z., 93 (1966), 171-188.

[8] O. T. O'Meara, Introduction to Quadratic Forms, Springer, New York, 1963. 
[9] C. Riehm, On the integral representations of quadratic forms over local fields, Amer. J. Math., 86 (1964), 25-62.

[10] Fei Xu, A remark on spinor norms of local integral rotations, I, Pacific J. Math., 136, No. 1 (1989), 81-84.

Received April 13, 1990 and in revised form October 16, 1991.

NANKai INSTitute of Mathematics

WEIJIN RoAD 94, TianJin 300071

People's Republic of ChinA 


\title{
PACIFIC JOURNAL OF MATHEMATICS
}

Founded by

\author{
E. F. Beckenbach (1906-1982) F. Wolf (1904-1989)
}

\section{EDITORS}

V. S. VARADARAJAN

(Managing Editor)

University of California

Los Angeles, CA 90024-1555

vsv@math.ucla.edu

Herbert Clemens

University of Utah

Salt Lake City, UT 84112

clemens@math.utah.edu

F. Michael Christ

University of California

Los Angeles, CA 90024-1555

christ@math.ucla.edu

THOMAS ENRIGHT

University of California, San Diego

La Jolla, CA 92093

tenright@ucsd.edu
Nicholas ERcolani

University of Arizona

Tucson, AZ 85721

ercolani@math.arizona.edu

R. FInN

Stanford University

Stanford, CA 94305

finn@gauss.stanford.edu

VAughan F. R. Jones

University of California

Berkeley, CA 94720

vfr@math.berkeley.edu

STEVEN KERCKHOFF

Stanford University

Stanford, CA 94305

spk@gauss.stanford.edu

\section{C. MOORE}

University of California

Berkeley, CA 94720

MARTIN SCHARLEMANN

University of California

Santa Barbara, CA 93106

mgscharl@henri.ucsb.edu

Harold Stark

University of California, San Diego La Jolla, CA 92093

\section{SUPPORTING INSTITUTIONS}

UNIVERSITY OF ARIZONA

UNIVERSITY OF BRITISH COLUMBIA

CALIFORNIA INSTITUTE OF TECHNOLOGY

UNIVERSITY OF CALIFORNIA

MONTANA STATE UNIVERSITY

UNIVERSITY OF NEVADA, RENO

NEW MEXICO STATE UNIVERSITY

OREGON STATE UNIVERSITY
UNIVERSITY OF OREGON

UNIVERSITY OF SOUTHERN CALIFORNIA

STANFORD UNIVERSITY

UNIVERSITY OF HAWAII

UNIVERSITY OF TOKYO

UNIVERSITY OF UTAH

WASHINGTON STATE UNIVERSITY

UNIVERSITY OF WASHINGTON 


\section{PACIFIC JOURNAL OF MATHEMATICS}

Volume $157 \quad$ No. $1 \quad$ January 1993

Permutation enumeration symmetric functions, and unimodality

FRANCESCO BRENTI

On the analytic reflection of a minimal surface

JAIGYOUNG CHOE

Contractive zero-divisors in Bergman spaces

Peter Larkin DUREn, DMitry Khavinson, Harold SEymour

SHAPIRO and CARL SUNDBERG

On the ideal structure of positive, eventually compact linear operators on

Banach lattices

RUEY-JEN JANG and HAROLD DEAN VICTORY, JR.

A note on the set of periods for Klein bottle maps

JAUME LLIBRE

Asymptotic expansion at a corner for the capillary problem: the singular case

\section{ERICH MIERSEMANN}

A state model for the multivariable Alexander polynomial

JUN MURAKAMI

Free Banach-Lie algebras, couniversal Banach-Lie groups, and more

Vladimir G. Pestov

Four manifold topology and groups of polynomial growth

RICHARD ANDREW STONG

A remark on Leray's inequality

AKIRA TAKESHITA

$A_{\infty}$ and the Green function

JANG-MEI GLORIA WU

Integral spinor norms in dyadic local fields. I 